In der Rubrik „Literatur kompakt" werden die wichtigsten Originalarbeiten aus der internationalen Fachliteratur referiert.

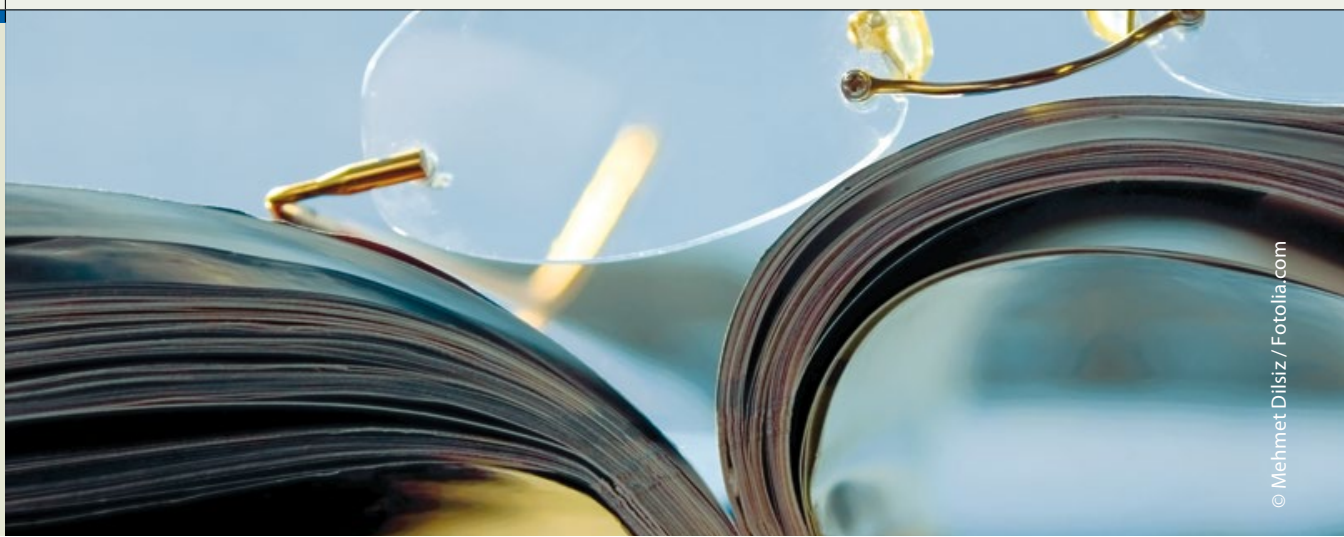

\section{Histaminarme Diät gegen chronische Urtikaria}

\section{Kann man durch den Verzicht auf stark histaminhaltige Lebensmittel die Symptome einer chronisch spontanen Urtikaria bessern? In einer deutschen Studie funktionierte das Rezept.}

E in Zusammenhang zwischen chroni- scher Urtikaria und Histaminaufnahme wird schon länger vermutet. In Studien wurden bei betroffenen Patienten Abweichungen im Histaminstoffwechsel und eine Induzierbarkeit von Attacken durch intraduodenale Histamingabe beobachtet. Zudem berichten viele Patienten über eine Verschlechterung ihrer Beschwerden nach dem Genuss von Rotwein, Hartkäse oder anderen histaminreichen Lebensmitteln. Ihre subjektive Einschätzung erhält nun Unterstützung durch eine aktuelle Studie mit 56 Patienten mit chronisch spontaner Urtikaria (CsU), die von gastroin- testinalen Beschwerden begleitet war. Im Rahmen der Studie wurden sie gebeten, sich mindestens drei Wochen lang gemäß einer Liste mit erlaubten Lebensmitteln zu ernähren. Ihre Ernährung ebenso wie die Aktivität der Urtikaria gemäß Urticaria Activity Score (UAS, 0-6 Punkte, höhere Werte entsprechen einem höheren Schweregrad) sollten sie in einem Tagebuch dokumentieren.

Die Summe der UAS-Werte an den vier Tagen vor Beginn der Intervention (UAS4) betrug im Mittel 9,05; sie reduzierte sich auf 4,23 an den letzten vier Tagen der Diät. Allerdings profitierten nicht alle Patienten: Bei neun (16\%) wurde kei-

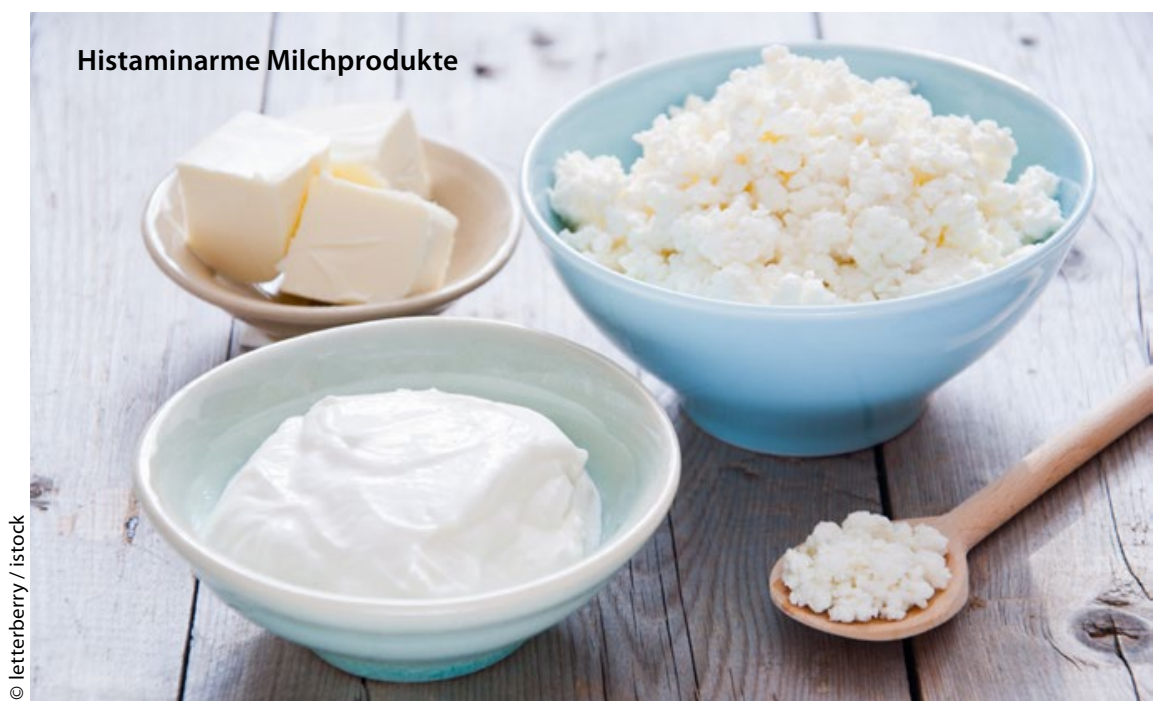

ne Veränderung und bei fünf (9\%) sogar eine Zunahme der Urtikariaaktivität festgestellt. Den primären Studienendpunkt, eine Verbesserung von mindestens drei Punkten im UAS4, erreichten 34 Patienten (61\%). Im Mittel war der UAS4 bei ihnen um 8,59 Punkte zurückgegangen. Ähnlich stark (-8,58 Punkte) war der Effekt in der Gruppe von Patienten, die initial einen UAS $4 \geq 9$ hatten.

Die Lebensqualität der Patienten verbesserte sich statistisch signifikant um 5,46 Punkte im Urtikaria-spezifischen Fragebogen CU-Q(2)oL. Im Dermatological Life Quality Instrument Questionnaire (DLQI) wurde das Signifikanzniveau dagegen verfehlt. Der Rückgang der Urtikariasymptome spiegelte sich auch in der Einnahme von Antihistaminika wider: Sie ging durchschnittlich um fast eine Tablette $(0,93)$ pro Tag zurück. Auf die Aktivität des histaminabbauenden Enzyms Diaminoxidase (DAO) hatte die Ernährungsumstellung keinen Einfluss.

Fazit: Unter einer histaminarmen Ernährung kam es bei den CsU-Patienten zu einem Rückgang der Symptome und einer Besserung der Lebensqualität. Zwar lässt sich aufgrund des fehlenden Vergleichs ein gewisser Placeboeffekt der Intervention nicht ausschließen. Die Studienautoren gehen aber davon aus, dass der Effekt im Wesentlichen auf den Histaminverzicht zurückzuführen ist. Die Maßnahme könne für eine Dauer von drei bis vier Wochen empfohlen werden, um Symptome und Antihistaminikaverbrauch zu reduzieren.

Dr. Beate Schumacher

Wagner N. et al. A Popular myth - low-histamine diet improves chronic spontaneous urticaria fact or fiction? J Eur Acad Dermatol Venereol 2017; 31:650-5 